\title{
Optical 3D In-Vivo Skin Imaging for Topographical Quantitative Assessment of Cosmetic and Medical Treatments
}

\author{
Christian BENDEROTH, Rolf HAINICH \\ GFMesstechnik GmbH*, Teltow, Germany
}

\begin{abstract}
The acquisition and evaluation of 3D data of human skin surfaces is an important aspect of both medical and cosmetic dermatology investigations. Accurate knowledge of skin surface topology is an important aid for accurate diagnoses, therapeutic decisions and evaluation of medical or cosmetic treatment progress.

Previously, 3D profile recognition of skin surfaces was mostly implemented through making skin impressions (replicas) and measuring them with tactile scanning devices. In contrast, the current state of technology is characterized by direct 3D in-vivo measurement.

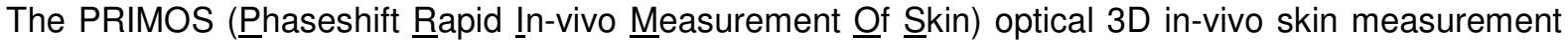
device uses fringe projection based on micro mirror display devices from Texas Instruments for optical measurement. The intensive R\&D work by Beiersdorf AG (Germany) and GFMesstechnik GmbH (Germany) has resulted in today's generation of optical 3D in vivo skin measuring systems.
\end{abstract}

\section{Introduction}

Since the first PRIMOS 3D measuring device was introduced in 1999, much has changed in the field of camera, display and computing technology. PRIMOS systems have taken advantage of this progress and become more powerful, smaller, flexible and cost-effective. Used in numerous clinical trials, cosmetic studies and medical assessments, PRIMOS has become a standard for optical 3D measurement in order to obtain skin topography with a high reproducibility and meaning. A major benefit of non contact in-vivo measurement is the objectivity of its measurements.

The number of applications and users of skin measurement has greatly increased. The quantitative determination of micro structures (such as skin roughness) and larger structures (such as wrinkles) is still the most important and probably the most frequently performed type of investigation in the field of cosmetics [1]. Nowadays, most practical dermatologists are expected to prove their quality.

In this area, facial wrinkles (ie. periorbital and perioral wrinkles, Glabella, forehead or nasiolabia folds, etc.) are major study subjects. Studies featuring these areas have been performed with the application of cosmetics, fillers, laser treatments and Botox.

In the field of medical treatment, PRIMOS systems are used to measure wound and scar tissue. PRIMOS systems can document the accuracy and reliability of pharmaceutical products or laser treatments on wound and scar healing. Previously, the gold standard for quantification of wounds and scars has been very subjective. Now, research labs and practical physicians worldwide are interested in an objective measurement method.

\footnotetext{
* GFMesstechnik GmbH, Warthestrasse 21, Teltow, Germany; www.gfm3d.com
} 


\section{PRIMOS optical 3D skin measurement system}

\subsection{Technology}

Unlike conventional fringe projection systems, the optical 3D skin measuring system PRIMOS (Phaseshift Rapid In vivo Measurement Of Skin) uses a digital fringe generation. This projection system is based on digital micro mirror arrays, developed and perfected by Texas Instruments during the 1990s [2]. Using digital micro mirror light projection offers a number of advantages, especially short exposure times resulting from the very high and pixel wise controllable light intensity of the projector $[3,4]$.

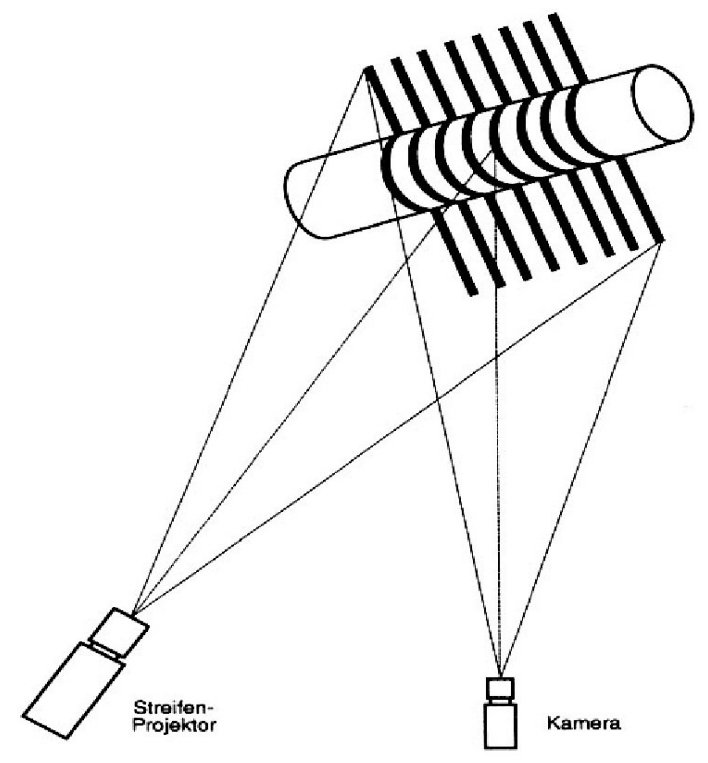

Fig. 1 Working principle of the PRIMOS optical 3D in vivo skin measuring device

As shown in fig. 1, a parallel stripe pattern is projected on the skin surface and recorded by means of an optical lens and the CCD image sensor of an electronic camera.

3D information is obtained, as even the slightest height differences on the skin surface cause an apparent deflection of the projected stripes as seen from the camera. These deflections affect the skin profile. Fig. 2 and 3 show typical stripe patterns for an inner arm surface and an eye corner (crow's feet). These patterns are recorded and digitized by the CCD camera and sent to a measuring and analysis computer.

The analysis uses highly sophisticated and precise mathematical algorithms originally developed for advanced measurement of optical and fine mechanical components. PRIMOS uses these algorithms to obtain a highly accurate 3D profile of the skin surface.

\subsection{Fringe projection based on DLP technology}

Along with lasers, scanning optical 3D measurements with structured light and/or projected fringe is the most popular method used in life sciences.

The basic principles of the projected fringe method are based on the real-time interferometry and triangulation technology frequently used worldwide. There are a number of different technologies for fringe projection and data acquisition in order to obtain a 3D point cloud of a measured object. Different options for generation of the projected fringes include: DLP, LCD, fixed gratings, frequency modulated gratings, etc. Recently, the DMD-based DLP technology has gained respect compared to other fringe projection measuring technologies. 


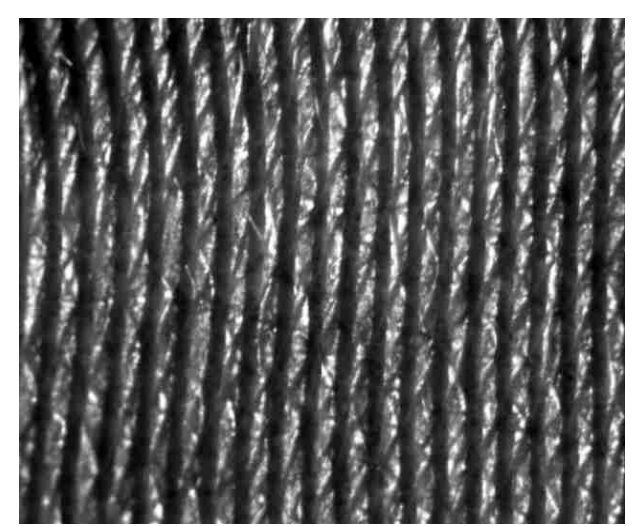

Fig. 2: Projected fringes, on lower arm

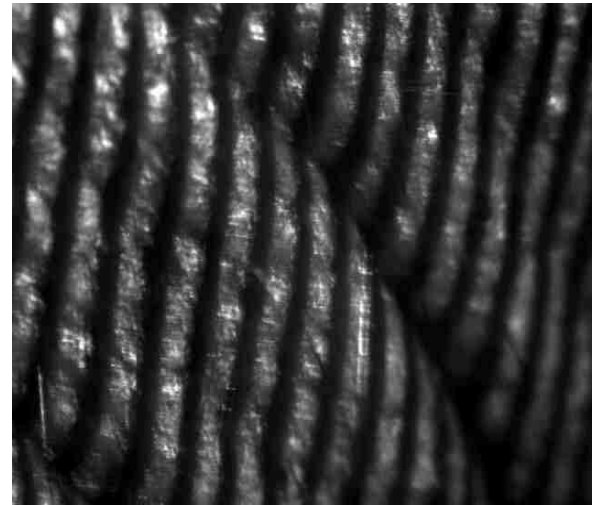

Fig. 3: Projected fringes, eye corner

\subsection{General basics}

The digital stripe projection used for the PRIMOS projection device works on the basis of micro mirror projection units (Digital Micromirror Devices - DMD ${ }^{\mathrm{TM}}$ ), which were developed and introduced by Texas Instruments Inc., U.S.A. [9, 10]. Using the DMD solution, Texas Instruments has opened up a completely new concept of digital light projection.

A DMD chip includes up to several million micro mirrors arranged in a square array. Each micro mirror can be tilted into an "on" or "off" position by electrostatic force generated by a CMOS chip structure underneath the micro mirror layer. The entire device is manufactured from a single, mono-crystalline silicon wafer by masking, doping and etching, a complex process that requires up to 43 steps.
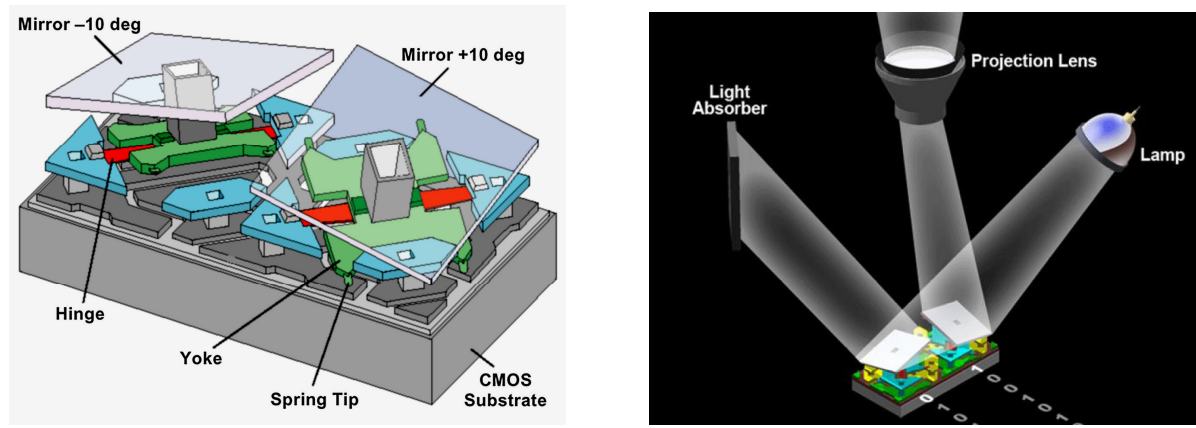

Fig.4: Micro mirrors, and mechanical design and pixels in "on" / "off" state
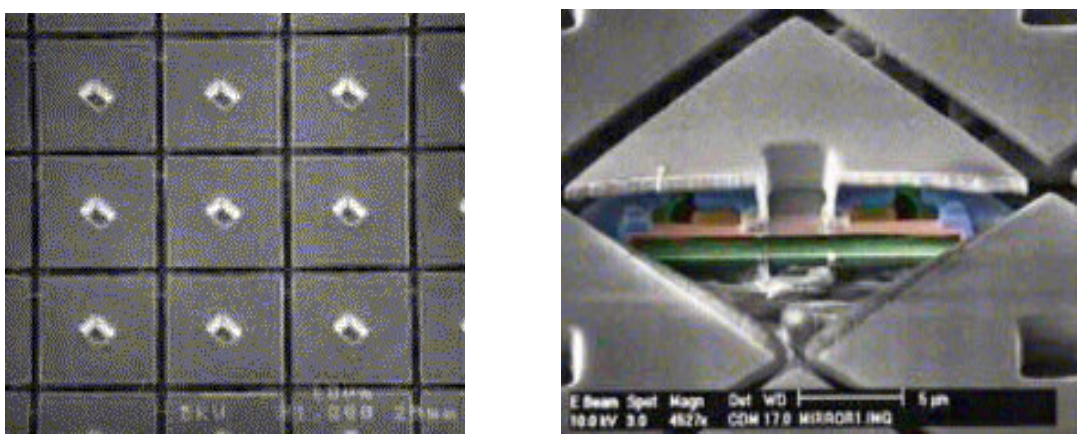

Fig.5: An REM image of micromirrors and a single micromirror 
By remaining in the "on" position for different times during a video frame, a sequence of binary images with an intensity range from 0 to 255 ( 8 bit grey scale) is produced. For example, if the grey value of 128 is to be produced, the respective micro mirrors directed at this grey value are kept in the "off" position for half of the video frame and in the "on" position for the other half.

The digital principle of micro mirror projection units makes a series of essential advantages possible for optical measurement technology, including:

\section{- High light performance}

The hidden mirror hinge realized in $\mathrm{DMD}^{\mathrm{TM}}$ enlarges the portion of the active mirror area to $90 \%$. In practice, due to losses from scattering, about $80 \%$ of the light introduced by the lamp will be reflected. Compared to that, the fill factor will be around $50 \%$ in the case of transmission LCD projectors, due to the necessary control electronics.

- High image contrast

Besides increased light performance, the high fill factor also improves the signal/noise ratio in the stripe patterns, because the portion of information-free gaps between the square pixels only amounts to $10 \%$. Together with the good reflection properties of the DMDTM, an excellent image contrast (> $50: 1)$ can thus be achieved.

\section{- Precise pixel projection}

The evenness of the micro mirror array produced by semiconductor technology guarantees the precision and stability of the projection direction for each pixel, which is crucial for measurement technology purposes. According to the DLPTM principle, each point is represented completely independent from its neighboring pixel, so that no spatial noise is generated. Short switching times let the projected stripes stay free of artifacts, even at fast changes.

- Exact reproducibility

The whole range of grey values is subject to digital control of the "on" and "off" times and depends in no way on material properties, saturation effects or the environmental temperature. The projected light intensities are therefore exactly reproducible and stable, which is advantageous for the respective measurement process.

\section{- Perfect color convergence}

The respective color components reach the projected image point by the same optical path, sequentially. That results in perfect convergence. Every color value can be set precisely by digital control.

\section{- Perfect linearity}

For application in stripe projection technology, micro mirror projectors offer the possibility, by exact intensity control due to the digital modulation, to generate exact sinus-shaped intensity distributions of the projected stripe patterns. Non-linearity in the intensity curves of the projected stripe patterns are, as a rule, responsible for systematic errors in the measurement result with other projection techniques. 


\subsection{Requirements for using a 3D scanner for medical and cosmetic skin measurement}

Capturing objective measurement data on living skin requires different parameters in order to gain high quality, reproducible data.

The vertical and lateral resolution of a stripe projection measurement device such as the PRIMOS system is essentially determined by

- The field of view (FOV) used

- The number of pixels of the recording camera

- The accuracy of the determination of the smallest stripe deflections that can still be processed.

To enable users to take advantage of the PRIMOS system's wide variety of different uses and to achieve optimal conditions for their respective applications, the sensors are available in different configurations. Beside the essential parameters, other considerations also have to be taken into account:

\section{Attention to eye safety}

DLP technology allows projecting light in different spectra. The regulation of light exposure when scanning a person is limited by safety rules [8]. In the European international standard EC 608251:1993 [4] with the corresponding national DIN EN 60825-1:2003-10 (VDE 0837), standard [7] and the addition from the BGIA.[6] the exposition limit values are defined. There is no additional specific standard for LED light sources since they belong to IEC 60825 [8].

Regarding this standard, there are different safety classes depending on the limited value for the exposure time. In order to scan human faces, the class $1 \mathrm{M}$ becomes a fundamental value. Class $1 \mathrm{M}$ products produce either a highly divergent beam or a large diameter beam. Therefore, only a small part of the whole light can enter the eye.

\section{Accuracy vs. measurement or capturing time}

The measurement time with fringe projection depends upon the number of different light patterns projected in a measuring sequence. On the other hand, measurement noise decreases and the robustness of the acquired data increase with the number of patterns. Another factor is choosing the best combination of camera parameters. Accuracy and capturing time for the imaging module can contradict each other. The density of captured measurement points depends upon the physical resolution of the camera. Increasing the camera resolution reduces the frame rate that can be transmitted or computed per second. PRIMOS systems are designed to run with a pattern rate of $60 \mathrm{~Hz}$. This allows for a measurement time down to $68 \mathrm{~ms}$, when using a sequence of 4 patterns.

\section{No influence of different lighting conditions}

The internal light source of the scanning system must be as strong as possible, reducing influences of arbitrary surrounding light sources. Unfortunately this conflicts with the requirement to create an eyesafe instrument. In order to comply with eye safety, the internal light source and optics must take into account the above-mentioned regulations.

\section{Using polarizer}

Polarizer attached in front of projection and camera optics allow for a better separation between light directly reflected from the skin surface and light diffused in the upper skin layers. Such diffusion may influence the perception of stripe positions and result in certain height value errors. Light directly reflected from the upper surface will mainly keep its polarization, while light diffused in the skin will get depolarized. This way, the polarizer filters can achieve an additional attenuation of diffused light by about $50 \%$. 


\section{The optical in vivo measurement system PRIMOS}

Taking into account the basic requirements listed above, GFMesstechnik, Teltow, developed the PRIMOS optical 3D in vivo skin measurement device. The digital fringe projection technique is deployed as an optical measurement process. Today's generation of optical 3D in vivo skin measuring systems is available with different fields of view, from $18 \times 13 \mathrm{~mm}^{2}$ up to $500 \times 350 \mathrm{~mm}^{2}$. Most of the systems are also able to take the color texture of the measured area beside the $3 \mathrm{D}$ information of skin structure.

PRIMOS systems are mostly used within clinical trials and studies. Obtaining quantitative and qualitative evaluation of changes before and after a treatment remains a perfect superimposition of images in order to assess numeric results of improvement.

\subsection{Superimposition of 3D images}

The imaging process helps localize the same area of measurement after treatment, as a ghost image of the reference picture can be displayed as an overlay in the live video view.

Experimental studies have shown that recovery in real time video is not yet sufficient for highly precise measurements, particularly of the effect of cosmetics on skin smoothness or the course of eye wrinkles. In order to increase the precision of such measurements and to detect and assess the smallest structural differences, an additional software-supported recovery area has been developed. To carry out this highly precise matching process, a reference image and at least one matching image must be stated. The software matches the complete sequence and shows a perfect superimposition of all pictures after the matching process. The fine structure of the images will not be deformed during the matching process.

In order to determine the differences between the initial or reference state and the currently measured comparative state, two different approaches can be chosen either separately or concurrently. On one hand, as shown in Fig. 22, profile sections can be put in the two skin profiles which can automatically be positioned exactly at the same location, based on the conducted highly precise matching of the skin profiles. Fig. 23 and 24 show these profile courses for the reference and measured areas with and without polynomial correction. Especially in the measurement output in Fig. 23, where the macroshape of the skin surface profile was subtracted by polynomial correction, the differences between the two skin surfaces before and after the treatment are clearly visible.

\subsection{Recommended applications}

PRIMOS systems are used for various applications. All treatments (cosmetic or medical) that apply a change to topography of skin might be a measurable application for a PRIMOS system. The most frequent uses for the systems are:

- Measurement of eye wrinkles (e.g., crow's feet, forehead wrinkles, etc.)

- Measurement of skin roughness and microprofile of skin surface

- Measurement of wounds, scars, acne 


\subsubsection{Wrinkle measurement}

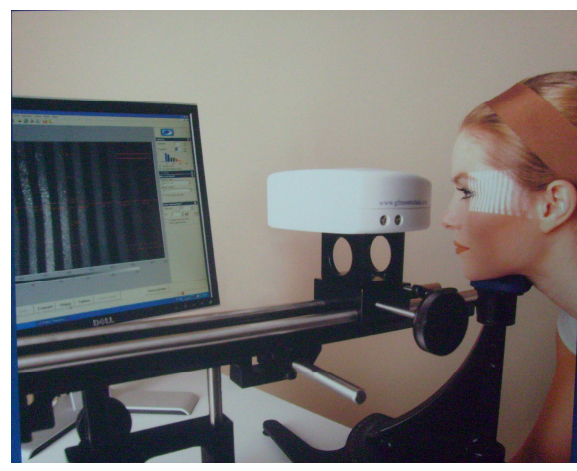

Fig. 6: Measurement of crow's feet

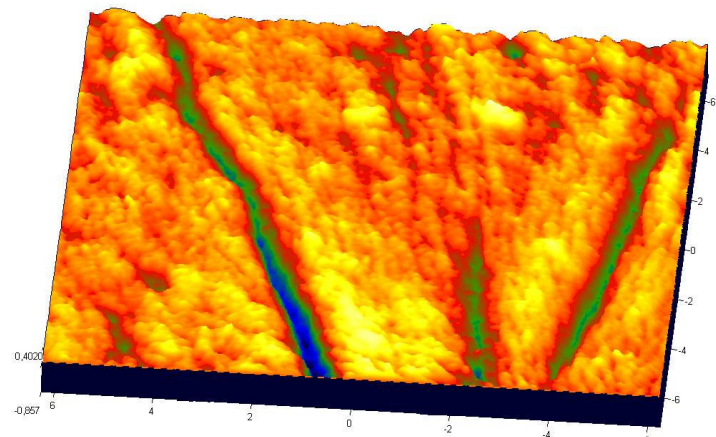

Fig. 7: False color image of crow's feet

The eyes are considered mirrors of the soul. People want to look young and are therefore trying to remove skin folds as the first signs of aging around the eyes.

To prove the effectiveness of various anti-aging products, the measurement of skin folds is essential in cosmetic and aesthetic medicine, as well as for the testing of cosmetic active substances. Fast, non-contact measurement of skin folds (e.g. crow's feet on the eye, the forehead, etc.) is possible using PRIMOS compact premium and PRIMOS lite. 3D measurement of the skin profile before and at different time points after a treatment (e.g. laser treatment), as well as their comparison using a precise matching option for area retrieval, enables the quantitative determination of skin fold changes and therefore a quantification of the treatment's success. Parameters of the changes are determination of the dimensions, the volume and the contour of the skin folds.

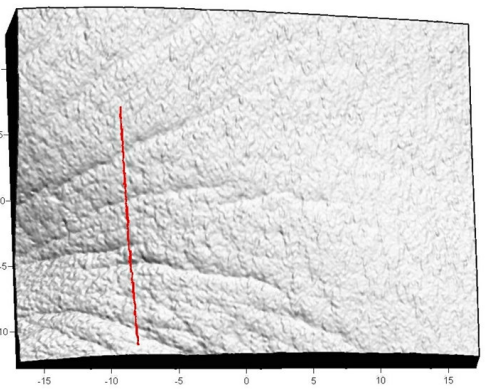

Fig. 8: 3D point cloud with sectional line

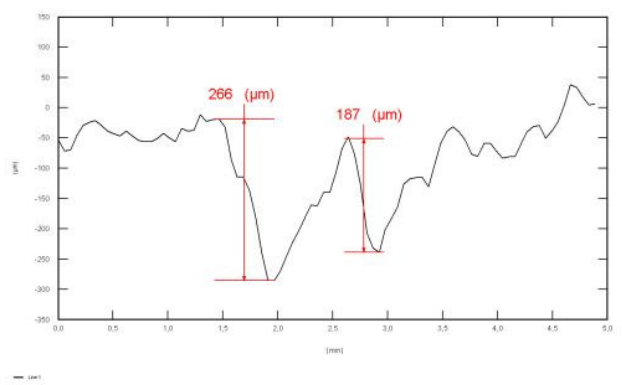

Fig. 9: 2D sectional line with depth measure

A new software was designed especially on the demand of automatic evaluation of wrinkles. 3D images are analyzed by an automatic algorithm that identifies wrinkles and gives you distribution of fine lines and wrinkles. Results are shown in a table with the value of average wrinkle depth, volume, max. depth and length. The calculation can be done in a database where hundreds of 3D images can be calculated at the same time.
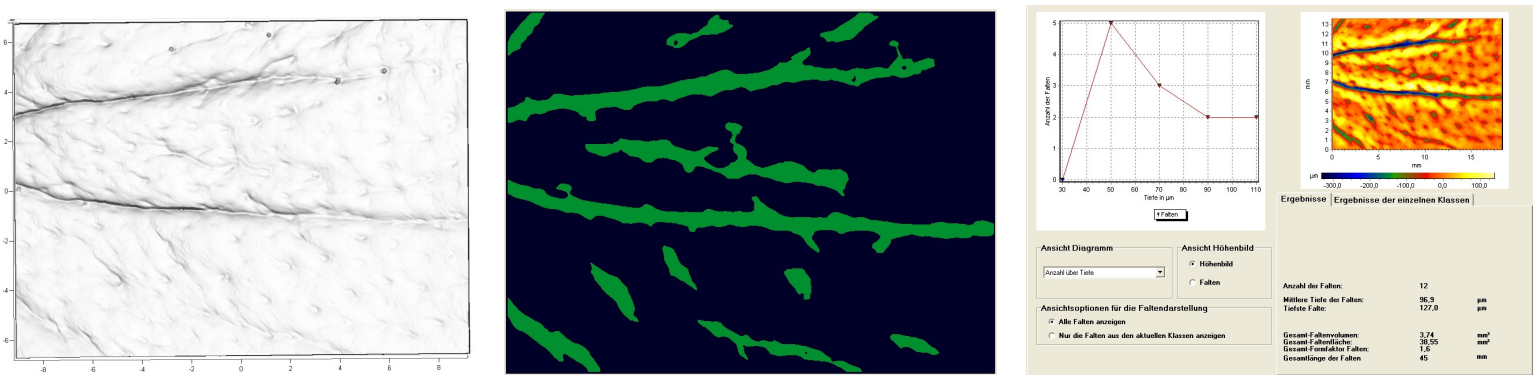

Fig. 10: 3D point cloud, automatic wrinkle detection and automatic calculation of wrinkle parameter 


\subsubsection{Skin roughness measurement}

Acquisition and evaluation of the surface of human skin is a task that has become a standard aspect of medical and cosmetic dermatology. In particular, for the validation of crèmes and medical active ingredients that affect skin roughness, optical in vivo measurements with PRIMOS offer a fast and efficient solution for objective results.

Using the PRIMOS compact high resolution, roughness measurements of an identical area can be made before and at different time points after a treatment with cosmetics or medical agents. The quantification of roughness can be accomplished by using line-, star- and area roughness parameters ( $R a, R z$...), which are calculated according to DIN 4288.

The main field of application of the PRIMOS compact is cosmetic and pharmaceutical research on active agents, cosmetic medicine and related applications.

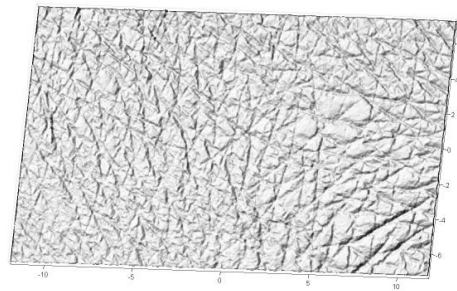

Fig. 11: Source 3D image of the skin microprofile
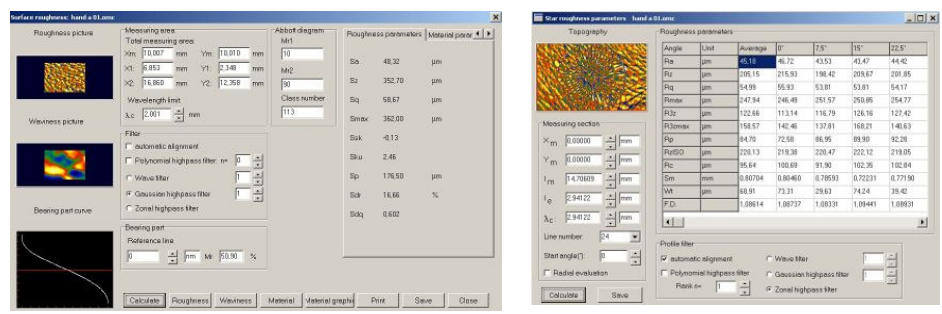

Fig. 12: Automatic evaluation of star or surface roughness parameters such as $R a, R z, \ldots$ Sa, Sz,...

\subsubsection{Wound and scar measurements}

The measurement and quantification of wounds or scars used to be difficult. Conventional replica methods were hardly usable. With PRIMOS measurement devices, fast, non-contact 3D in vivo measurement is possible.

The 3D measurement of a skin area before and at different time points after a treatment, as well as the computer-supported comparison of measured profiles, enables the quantitative determination of changes on wounds and scars and therefore control over the healing process. Non-contact measurement allows the acquisition of all changes in the skin contour like wounds, scars, pustules, welts or swells for objective evaluation. Changes in dimensions, volume or form can be determined fast and easily.

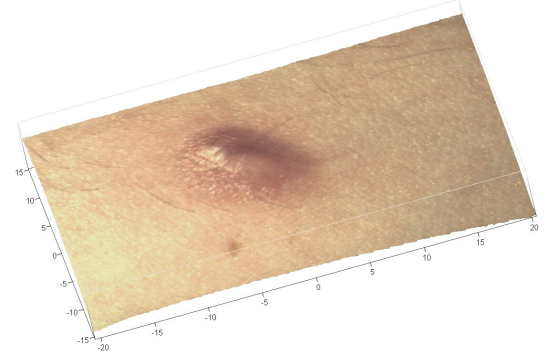

Fig. 13: Source 3D image of the brand scar

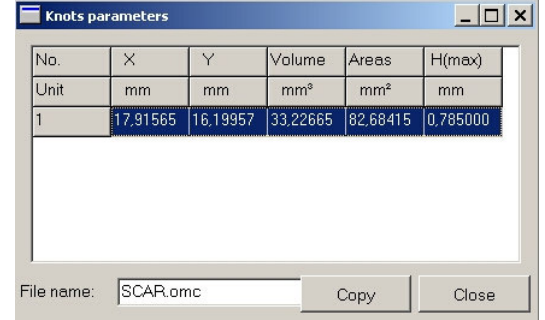

Fig. 14: Automatic determination of scar area and volume 


\section{PRIMOS systems for face and body: FaceSCAN 3D \& BodySCAN 3D}

The FaceSCAN and BodySCAN measuring device allows for fast 3D measurement in combination with the acquisition of colour textures. It can be used to capture an entire face with one single measurement in only $140 \mathrm{~ms}$. The use of the newest camera technology in combination with the digital $3 \mathrm{D}$ stripe projection provide objective measuring results for the documentation of surgical treatments and many other applications. The GFM concept shows a single scanner with a measurement field of $500 \times 400 \mathrm{~mm}^{2}$ what can be combined with up to three more single scanners in order to get a view up to $360^{\circ}$. For face measurement the field of view is slightly smaller and a combination of two scanners is recommended to get a full face 3D image. Comparative measurements can be taken on the
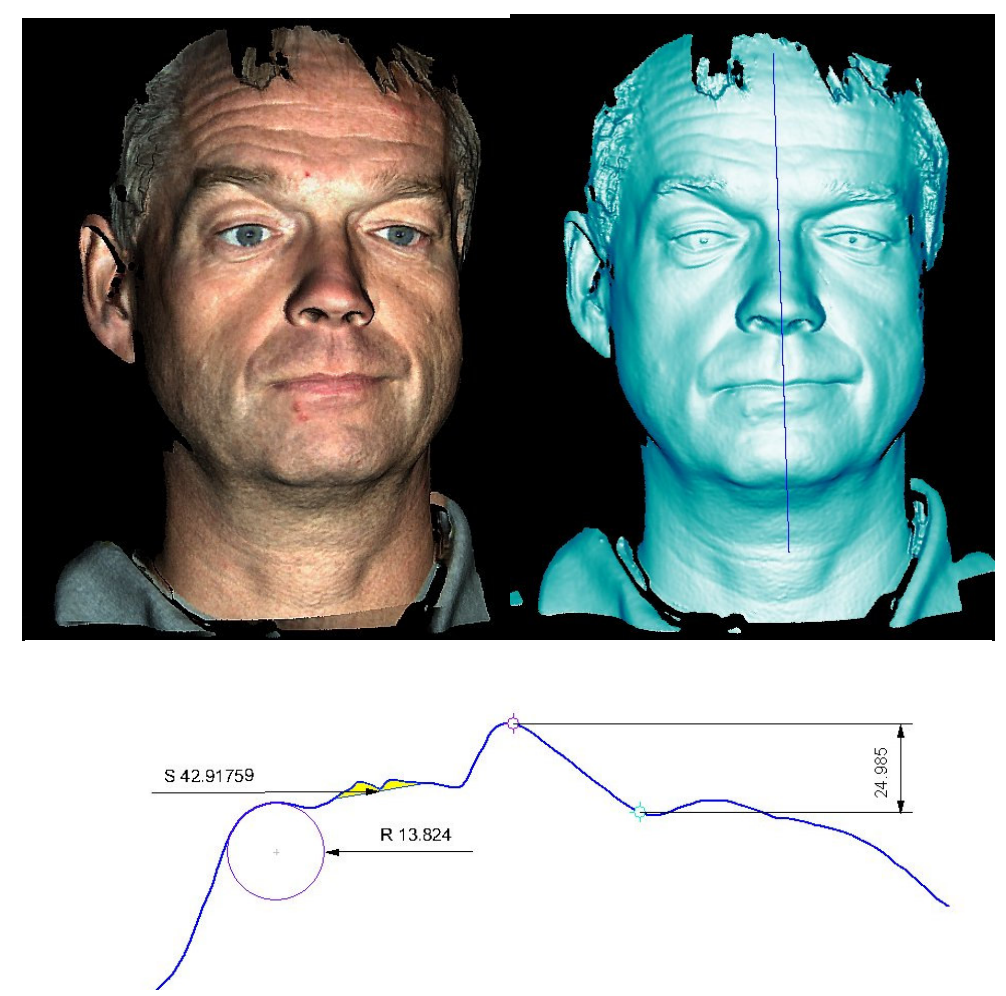

Fig. 15: 3D image of full face with color texture and point cload with sectional line. The cross section can be taken with various measurements on its profile.

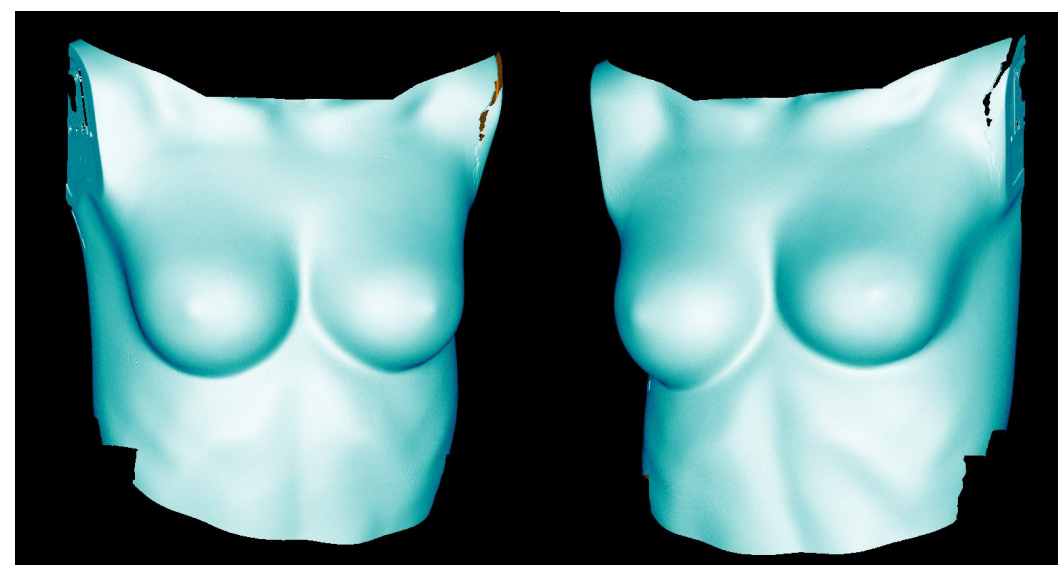

Fig.16: 3D image of breast area 


\section{Conclusion}

PRIMOS 3D systems provide an easy, objective and reproducible analysis of skin topography. With that important preconditions for validations of cosmetic products and treatments are given. Within the context of many studies and presentations the PRIMOS systems are used as measurement method. PRIMOS is used worldwide by cosmetic companies, testing institutes, dermatology departments and universities. The results of measurement are not restricted by the use for cosmetics. More and more dermatology application becomes important applications for 3D topography in order to diagnose skin changes caused by illness.

\section{References}

[1] Jaspers S. Fringe projection for in vivo topometry. In: KPWilhelm, EBeradesca, PElsner, HIMaibach, eds. Bioengineering of the Skin: ISBN 9780849338175

[2] Xiong Jiang, Ezra Rosen, Thomas Zeffiro, John VanMeter, Volker Blanz, Maximilian Riesenhuber, "Evaluation of a Shape-Based Model of Human Face Discrimination Using fMRI and Behavioral Techniques"

[3] Hornbeck, L. J.: Digital Light Processing ${ }^{\mathrm{TM}}$ and MEMS: Timely Convergence for Bright Future, Micromachining and Microfabrication '95, Austin/TX, USA, 1995

[4] Frankowski, G.: The ODS 800 - a new projection unit for optical metrology; Proceedings of Fringe '97; 3rd Int. Workshop on "Automatic Processing of Fringe Patterns"; Bremen, Sept. 15 17,1997

[5] Frankowski, G., Chen, M., Huth, T.: Real-time 3D Shape Measurement with Digital Stripe Projection by Texas Instruments Micromirror Devices DMD ${ }^{\mathrm{TM}}$;

[6] Monk, D. W. and Gale, R.: The Digital Micromirror Device for Projection Display (invited paper), Microelectronic Engineering, Vol. 27, pp. 489-493 (1995)

[7] Yoder, L. A.: The Digital Display Technology of the Future, INFOCOM '97, 5-7, Los Angeles, CA; USA, June 1997

[8] EC 60825-1:1993 + A2:2001-01, Safety of laser products - Part 1: Equipment classification, requirements and user's guide, 2001.

[9] Expositionsgrenzwerte für künstiliche optische Strahlung, Berufsgenossenschaftliche Information für Sicherheit und Gesundheit bei der Arbeit. Oktober 2004.

[10] DIN EN 60825-1:2003-10 (VDE 0837), Sicherheit von Lasereinrichtungen - Teil 1: Klassifizierung von Anlagen, Anforderungen und Benutzer-Richtlinien (IEC 60825-1:1993 + A2:2001), November 2001. 